\title{
Mesh-free Hamiltonian implementation of two dimensional Darwin model
}

Lorenzo Siddi, Giovanni Lapenta, and Paul Gibbon

Citation: Physics of Plasmas 24, 082103 (2017); doi: 10.1063/1.4994705

View online: https://doi.org/10.1063/1.4994705

View Table of Contents: http://aip.scitation.org/toc/php/24/8

Published by the American Institute of Physics

\section{Articles you may be interested in}

Variational principle for the parallel-symplectic representation of electromagnetic gyrokinetic theory

Physics of Plasmas 24, 081201 (2017); 10.1063/1.4997484

Magnetohydrodynamic motion of a two-fluid plasma

Physics of Plasmas 24, 082104 (2017); 10.1063/1.4994068

Gyrokinetic particle simulations of the effects of compressional magnetic perturbations on drift-Alfvenic instabilities in tokamaks

Physics of Plasmas 24, 081205 (2017); 10.1063/1.4997788

Centrifugal instability in the regime of fast rotation

Physics of Plasmas 24, 082102 (2017); 10.1063/1.4994546

Mitigation of the cancellation problem in the gyrokinetic particle-in-cell simulations of global electromagnetic modes

Physics of Plasmas 24, 081206 (2017); 10.1063/1.4997540

Extreme driven ion acoustic waves

Physics of Plasmas 24, 082106 (2017); 10.1063/1.4986031

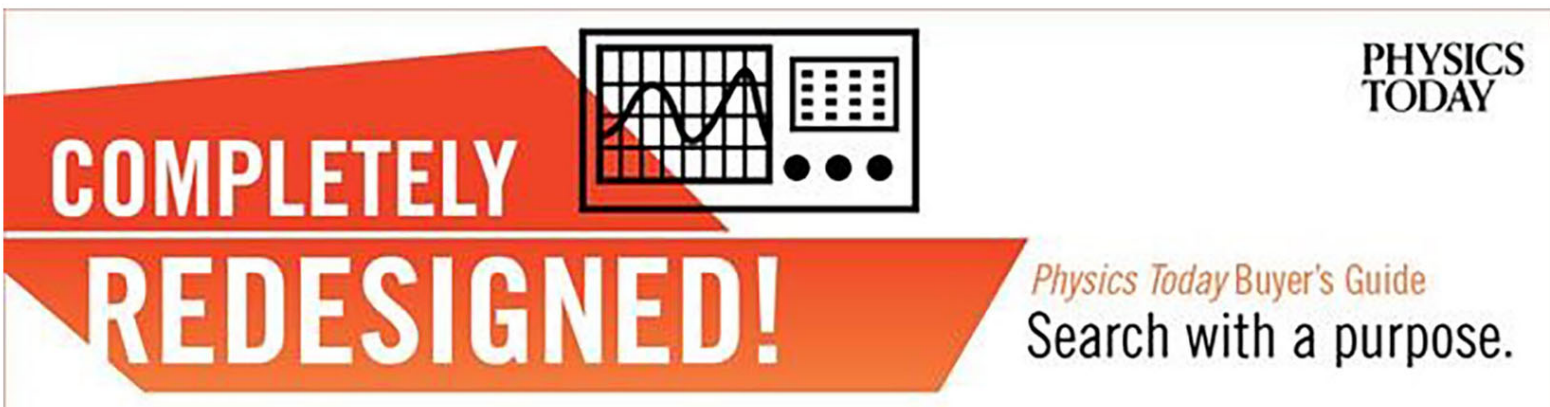




\title{
Mesh-free Hamiltonian implementation of two dimensional Darwin model
}

\author{
Lorenzo Siddi, ${ }^{1, a)}$ Giovanni Lapenta, ${ }^{1, b)}$ and Paul Gibbon ${ }^{1,2, c)}$ \\ ${ }^{1}$ Center for mathematical Plasma Astrophysics, Department of Mathematics, K.U. Leuven, Celestijnenlaan \\ 200B, B-3001 Leuven, Belgium \\ ${ }^{2} J$ ülich Supercomputing Centre (JSC), Forschungszentrum Jülich GmbH, Wilhelm-Johnen-Straße, 52425 \\ Jülich, Germany
}

(Received 8 February 2017; accepted 6 July 2017; published online 20 July 2017)

\begin{abstract}
A new approach to Darwin or magnetoinductive plasma simulation is presented, which combines a mesh-free field solver with a robust time-integration scheme avoiding numerical divergence errors in the solenoidal field components. The mesh-free formulation employs an efficient parallel Barnes-Hut tree algorithm to speed up the computation of fields summed directly from the particles, avoiding the necessity of divergence cleaning procedures typically required by particle-incell methods. The time-integration scheme employs a Hamiltonian formulation of the Lorentz force, circumventing the development of violent numerical instabilities associated with time differentiation of the vector potential. It is shown that a semi-implicit scheme converges rapidly and is robust to further numerical instabilities which can develop from a dominant contribution of the vector potential to the canonical momenta. The model is validated by various static and dynamic benchmark tests, including a simulation of the Weibel-like filamentation instability in beam-plasma interactions. Published by AIP Publishing. [http://dx.doi.org/10.1063/1.4994705]
\end{abstract}

\section{INTRODUCTION}

The full electromagnetic description of plasma is a computationally intensive endeavor governed by the hyperbolic character of Maxwell's equations, and the associated need to resolve light waves on a spatial grid. However, for scenarios dominated by slowly evolving processes where electromagnetic wave propagation can be neglected, Maxwell's equations can be simplified into a form more suitable for following collective phenomena on longer time scales. The Darwin or magnetoinductive limit of Maxwell's equations is one such approach which achieves this compromise, and is obtained by neglecting the transversal (otherwise known as solenoidal, or divergence-free) contribution of the displacement current in Ampère's law. This omission turns the characteristic hyperbolic structure of Maxwell's equations into an elliptic system, such that the Courant-Friedrichs-Lewy (CFL) condition for the time step, namely $\Delta t<\Delta x / c$, where $\Delta x$ and $c$ are the grid space and the speed of light, respectively, does not need to be satisfied, leaving more scope for using larger time steps.

Darwin models are unfortunately not so simple to implement. To date, many authors have developed and worked with Darwin particle-in-cell (PIC) simulations with mixed success. In their pioneering paper, Nielsen \& Lewis ${ }^{1}$ showed that any attempt to derive the solenoidal field $\mathbf{E}^{\text {sol }}$ via the time derivative of the vector potential (Lagrangian derivative) is not consistent with the Darwin limit and causes violent numerical instabilities. In fact, a direct approach to the evaluation of $\mathbf{E}^{\text {sol }}$ reintroduces electromagnetic radiation through mutually inductive currents which in principle have been neglected. This obstacle can be overcome by deploying moments of the Vlasov equation to

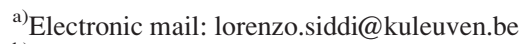

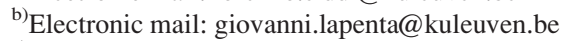

${ }^{\mathrm{c}}$ Electronic mail: p.gibbon@fz-juelich.de
}

eliminate explicit time-derivatives, which yields instead a elliptical equation for $\mathbf{E}^{s o l}$, the solution of which needs additional "divergence cleaning" techniques to enforce the Coulomb gauge condition $\nabla \cdot \mathbf{A}^{2,3}$ Other authors have proposed Vlasov-Darwin models, ${ }^{4,5}$ including a charge-conserving implementation, ${ }^{6}$ which present additional challenges for multi-dimensional problems with complex boundary conditions.

Recently, Masek \& Gibbon ${ }^{7}$ proposed a proof-of-principle mesh-free formulation of a three-dimensional Darwin model which behaved satisfactorily for simple test cases, but ran into difficulties for more general scenarios in which arbitrarily strong vector potentials were permitted. Nevertheless, the mesh-free approach does offer some significant advantages compared to traditional grid-based codes, which motivate the present quest for a more robust algorithm. For example, in high-density plasmas, Coulomb collisions become significant, which in principle can be included directly through a meshfree approach, rather than via an ad-hoc Monte-Carlo scattering operator as is common in PIC methods. For collisionless plasmas, the absence of a grid removes aliasing instabilities (and their associated numerical heating) by design, permitting the simulation of initially cold plasmas. A gridless method is also intrinsically adaptive and naturally offers more flexible boundary conditions, ${ }^{8}$ including, for example, truly open (vacuum) boundaries for modeling isolated systems.

As a first step towards the development of a robust three-dimensional code, we present a mesh-free two-dimensional Darwin formulation combined with a Hamiltonian integration time scheme. This approach circumvents the use of $\mathbf{E}^{\text {sol }}$ in the particle integrator, employing instead gradients of a divergence-free vector potential.

This paper is organized as follows: first, in Sec. II, the classical Darwin approximation is introduced. This serves as the basis for a new two-dimensional formulation proposed in Sec. III, including a special formulation of the scalar and 
vector potentials consistent with a finite-size particle density profile. We then discuss the direct solution of the Darwin field equations via a Barnes-Hut tree algorithm, permitting a rapid $O(N \log N)$ summation, just as for purely electrostatic systems. The integration scheme adopted is described in Sec. IV; it is a stable integrator, implicit in space and explicit in velocity. Finally, we validate the model with various benchmark tests. The first of these examines the accuracy of the field solver in a cylindrical plasma having uniform density. Dynamic tests on Langmuir waves and vacuum beam propagation validate the robustness and accuracy of the integration scheme. Finally, the well-known Weibel-like filamentation instability in high-density plasma is examined, comparing results with previous grid-based models.

\section{MAXWELL'S EQUATIONS}

In this section, we briefly recap on the formulation of Maxwell's equations according to the Darwin or magnetoinductive model, the description followed is the same as in previous works. ${ }^{1-3,7,9}$ A key procedure is the use of Helmholtz's theorem, which states that any vector can be decomposed into irrotational and solenoidal parts. For instance, the electric field $\mathbf{E}=\mathbf{E}^{i r r}+\mathbf{E}^{\text {sol }}$. Therefore, we can rewrite the Maxwell's equation as follows:

$$
\begin{gathered}
\nabla \cdot \mathbf{E}^{i r r}=4 \pi \rho, \\
\nabla \times \mathbf{E}^{s o l}=-\frac{1}{c} \frac{\partial \mathbf{B}}{\partial t}, \\
\nabla \cdot \mathbf{B}=0, \\
\nabla \times \mathbf{B}=\frac{4 \pi}{c} \mathbf{J}+\frac{1}{c} \frac{\partial \mathbf{E}^{i r r}}{\partial t}+\frac{1}{c} \frac{\partial \mathbf{E}^{s \rho t}}{\partial t} .
\end{gathered}
$$

The Darwin model neglects the solenoidal component of the displacement current in Ampère's Law and consequently presupposes that information is transmitted instantaneously. This is because the hyperbolic nature of Maxwell's equations is replaced by an elliptic system which neglects electromagnetic wave propagation. In fact, it is possible to rewrite Eqs. (1)-(4) in the Coulomb gauge $(\nabla \cdot \mathbf{A}=0)$ in the form of two Poisson-like equations

$$
\begin{gathered}
\nabla^{2} \varphi=-4 \pi \rho, \\
\nabla^{2} \mathbf{A}=-\frac{4 \pi}{c} \mathbf{J}^{\text {sol }},
\end{gathered}
$$

where the fields can be recovered from

$$
\begin{gathered}
\mathbf{E}=\mathbf{E}^{i r r}+\mathbf{E}^{s o l}, \\
\mathbf{E}^{i r r}=-\nabla \varphi, \\
\mathbf{J}^{\text {sol }}=\mathbf{J}+\frac{1}{4 \pi} \frac{\partial \mathbf{E}^{i r r}}{\partial t}=\mathbf{J}-\mathbf{J}^{i r r}, \\
\mathbf{B}=\nabla \times \mathbf{A} .
\end{gathered}
$$

It is worth noting here that in general, Amperè's law is not gauge invariant, ${ }^{9}$ a fact that will influence our formulation of the model vector potential in Sec. III.

\section{2D DARWIN MODEL FOR FINITE-SIZED PARTICLES}

In principle, particles in an N-body system can be treated as point charges ${ }^{10,11}$ and then modeled by a Dirac delta function. Numerically, this is impractical because it introduces divergences in the scalar and vector potentials, which become rapidly unmanageable for the integration scheme. We can circumvent this issue by employing a smooth particle profile, as frequently deployed in astrophysical models. ${ }^{12}$ Concretely, we need a simple particle's model so that we are able to analytically solve the Maxwell-Darwin system of equations. Therefore, the profile proposed is a radially symmetric shape function, ${ }^{13}$ defined as

$$
\begin{gathered}
S_{\varepsilon}(\mathbf{x})=\frac{\varepsilon^{2}}{\pi\left(\|\mathbf{x}\|^{2}+\varepsilon^{2}\right)^{2}}, \\
\lim _{\varepsilon \rightarrow 0} S_{\varepsilon}(\mathbf{x})=\delta(\mathbf{x}),
\end{gathered}
$$

where the parameter $\varepsilon$ is a smoothing parameter, which can be tuned to control the collisionality. The function $S_{\varepsilon}$ is chosen as the Plummer profile and it is straightforward to show that this function tends to Dirac delta function when $\varepsilon$ becomes zero. Higher-order shape functions are in principle possible, but make the subsequent derivation of the vector potential mathematically more complex. Previous experience with electrostatic mesh-free implementations ${ }^{14-16}$ has shown that the above particle profile can be deployed in both collisional and collisionless regimes, motivating the retention of this analytically convenient choice for the present Darwin model. For the collisionless systems of primary interest here, it suffice to ensure that $\varepsilon \gg \bar{a}$, where $\bar{a}$ is the mean interparticle spacing. In a statistical sense, $\varepsilon$ plays an analogous role to the grid spacing in PIC codes, which typically call for "many particles per cell or Debye length." Here, the analogy stops though: in our method, there is no requirement that $\varepsilon$ $\leq \lambda_{D}$ for preventing numerical heating.

In the following analysis, we will simplify the formulas with the substitution, $r_{i j}^{2}=\left\|\mathbf{x}_{i}-\mathbf{x}_{j}\right\|^{2}$.

We identify two sources in the coupled system 5-6, which are the density $\rho$ and the solenoidal part of the current density $\mathbf{J}^{\text {sol }}$. We first define the density

$$
\rho\left(\mathbf{x}_{i}\right)=\sum_{j \neq i} q_{j} S_{\varepsilon}\left(\mathbf{x}_{i}-\mathbf{x}_{j}\right)=\sum_{j \neq i} \frac{q_{j} \varepsilon^{2} / \pi}{\left(r_{i j}^{2}+\varepsilon^{2}\right)^{2}} .
$$

Substituting the definition of density in the continuity equation, the corresponding current density is, for a set of mobile particles, expressed by

$$
\mathbf{J}\left(\mathbf{x}_{i}\right)=\sum_{j \neq i} q_{j} \mathbf{v}_{j} S_{\varepsilon}\left(\mathbf{x}_{i}-\mathbf{x}_{j}\right)=\sum_{j \neq i} \frac{q_{j} \mathbf{v}_{j} \varepsilon^{2} / \pi}{\left(r_{i j}^{2}+\varepsilon^{2}\right)^{2}} .
$$

Our initial aim is to analytically solve the system 5-6 employing the Plummer profile in an unbound domain. This approach has two goals: the first is to develop an efficient method using open boundary conditions, and secondly to 
retain sufficient flexibility to include periodic boundary conditions. To proceed, we employ the Green's function method to solve the Poisson equation, which allows us to use an unbound domain without a particular issue.

In two dimensions, the Green's function is

$$
\mathcal{G}\left(\mathbf{x} ; \mathbf{x}^{\prime}\right)=\frac{1}{4 \pi} \log \left(\left\|\mathbf{x}-\mathbf{x}^{\prime}\right\|^{2}\right)+h,
$$

where $h$ is a harmonic function. First, we solve the following system:

$$
\begin{gathered}
\nabla^{2} \varphi=-4 \pi \rho, \\
\nabla^{2} \hat{\mathbf{A}}=-\frac{4 \pi}{c} \mathbf{J}, \\
\varphi\left(\mathbf{x}_{i}\right)=-4 \pi \rho\left(\mathbf{x}_{i}\right) * \mathcal{G}\left(\mathbf{x}_{i} ; \mathbf{x}^{\prime}\right)=-\sum_{j \neq i} q_{j} \log \left(r_{i j}^{2}+\varepsilon^{2}\right), \\
\hat{\mathbf{A}}\left(\mathbf{x}_{i}\right)=-\frac{4 \pi}{c} \mathbf{J}\left(\mathbf{x}_{i}\right) * \mathcal{G}\left(\mathbf{x}_{i} ; \mathbf{x}^{\prime}\right)=-\frac{1}{c} \sum_{j \neq i} q_{j} \mathbf{v}_{j} \log \left(r_{i j}^{2}+\varepsilon^{2}\right) .
\end{gathered}
$$

From these quantities, it is straightforward to write down the electric and magnetic vector fields

$$
\begin{gathered}
\mathbf{E}^{i r r}\left(\mathbf{x}_{i}\right)=-\nabla \varphi=2 \sum_{j \neq i} q_{j} \frac{\mathbf{x}_{i}-\mathbf{x}_{j}}{r_{i j}^{2}+\varepsilon^{2}}, \\
\mathbf{B}\left(\mathbf{x}_{i}\right)=\nabla \times \hat{\mathbf{A}}=-\frac{2}{c} \sum_{j \neq i} q_{j} \frac{\left(\mathbf{x}_{i}-\mathbf{x}_{j}\right) \times \mathbf{v}_{j}}{r_{i j}^{2}+\varepsilon^{2}} .
\end{gathered}
$$

We employ Â only for the sake of evaluation of $\mathbf{B}$. In fact, $\hat{\mathbf{A}}$ is not solenoidal and does not satisfy Eq. (6). Although the vector potential A satisfies a Poisson-like equation, the integration of its equation in two dimensions leads to diverging terms using the Green's function method. In fact, we can write the solenoidal current density ${ }^{7}$ as

$$
\mathbf{J}_{\text {sol }}=\frac{1}{4 \pi} \nabla \times \nabla \times\left(\mathbf{J}\left(\mathbf{x}_{i}\right) * \mathcal{G}\left(\mathbf{x}_{i} ; \mathbf{x}^{\prime}\right)\right) .
$$

Therefore, by inverting the Laplacian in Eq. (6), we obtain

$$
\mathbf{A}=-\frac{4 \pi}{c} \mathbf{J}_{s o l}\left(\mathbf{x}_{i}\right) * \mathcal{G}\left(\mathbf{x}_{i} ; \mathbf{x}^{\prime}\right)
$$

The double convolution $\mathcal{G}\left(\mathbf{x}_{i} ; \mathbf{x}^{\prime}\right) * \mathcal{G}\left(\mathbf{x}_{i} ; \mathbf{x}^{\prime}\right)$ in $\mathbb{R}^{2}$ yields diverging terms. To circumvent this issue, we propose an alternative method, which starts from the Coulomb gauge condition. In fact, since $\mathbf{A}$ is solenoidal, it must be a curl of some vector, so that $\nabla \cdot \mathbf{A}=0$. We make the following assumption:

$$
\mathbf{A}\left(\mathbf{x}_{i}\right)=\frac{1}{2 c} \sum_{j \neq i} q_{j} \nabla \times\left[f\left(r_{i j}^{2}\right)\left(\mathbf{x}_{i}-\mathbf{x}_{j}\right) \times \mathbf{v}_{j}\right] .
$$

Hence, the vector $\left(\mathbf{x}_{i}-\mathbf{x}_{j}\right) \times \mathbf{v}_{j}$ is parallel to $\mathbf{B}$, which has only the $\mathrm{z}$ component in two dimensions, it is mapped in the plane $x-y$ by applying the curl operator (Fig. 1). We assume $f$ to be a radially symmetric function. Since the other fields possess this property, it is reasonable to assume the same for

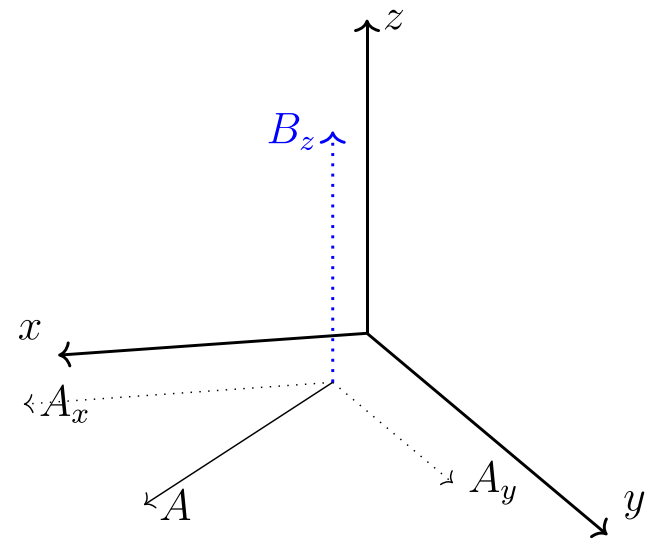

FIG. 1. Vector potential sketch, two-dimensional in both space and velocity (2D2V). Under this assumption, $\mathbf{B}=B_{z} \mathbf{k}=\nabla \times \mathbf{A}=\nabla \times\left(A_{x} \mathbf{i}+A_{y} \mathbf{j}\right)$.

A, which also simplifies its evaluation. In order that the Maxwell-Darwin limit is consistent, the curl of Eq. (24) must match Eq. (21), which yields a second order differential equation for $f$ and $r_{i j}$ as dependent and independent variables, respectively. These arguments lead to the following function satisfying the above-mentioned differential equation

$$
\begin{gathered}
f\left(r_{i j}^{2}\right)=2-\frac{\varepsilon^{2}}{r_{i j}^{2}} \log \left(\frac{r_{i j}^{2}}{\varepsilon^{2}}+1\right)-\log \left(\frac{r_{i j}^{2}}{\varepsilon^{2}}+1\right), \\
f^{\prime}\left(r_{i j}^{2}\right)=\frac{1}{r_{i j}^{2}}\left[\frac{\varepsilon^{2}}{r_{i j}^{2}} \log \left(\frac{r_{i j}^{2}}{\varepsilon^{2}}+1\right)-1\right] .
\end{gathered}
$$

Thus, the vector potential takes the form

$$
\begin{aligned}
\mathbf{A}\left(\mathbf{x}_{i}\right)= & \frac{1}{2 c} \sum_{j \neq i} q_{j} \nabla \times\left[f\left(r_{i j}^{2}\right)\left(\mathbf{x}_{i}-\mathbf{x}_{j}\right) \times \mathbf{v}_{j}\right] \\
= & \frac{1}{2 c} \sum_{j \neq i} q_{j} f\left(r_{i j}^{2}\right) \mathbf{v}_{j}+\frac{1}{c} \sum_{j \neq i} q_{j} f^{\prime}\left(r_{i j}^{2}\right) \frac{\mathbf{x}_{i}-\mathbf{x}_{j}}{\left\|\mathbf{x}_{i}-\mathbf{x}_{j}\right\|} \\
& \times\left[\mathbf{v}_{j} \times \frac{\mathbf{x}_{i}-\mathbf{x}_{j}}{\left\|\mathbf{x}_{i}-\mathbf{x}_{j}(t)\right\|}\right] .
\end{aligned}
$$

It is straightforward to verify that the vector potential Eq. (27) reverts to the (2D) point particle vector potential for $\varepsilon \rightarrow 0^{+}$

$$
\mathbf{A}_{\delta}\left(\mathbf{x}_{i}\right)=-\frac{1}{2 c} \sum_{j \neq i} q_{j}\left[\mathbf{v}_{j} \log \left(r_{i j}^{2}\right)+\left(\left(\mathbf{x}_{i}-\mathbf{x}_{j}\right) \cdot \mathbf{v}_{j}\right) \frac{\mathbf{x}_{i}-\mathbf{x}_{j}}{r_{i j}^{2}}\right] .
$$

It is worth mentioning that the choice of Eq. (25) has other important properties, in fact, it guarantees that $\mathbf{A}$ does not diverge when $r_{i j} \rightarrow 0^{+}$and the vector potential has indeed an upper bound

$$
\mathbf{A}\left(\mathbf{x}_{i}\right) \leq \frac{1}{2 c} N[1-2 \log \varepsilon] \max _{j}\left(q_{j} \mathbf{v}_{j}\right),
$$

where $N$ is the number of particles.

In many applications in plasma, a two-dimensional approach in space and three-dimensional in velocity is more realistic. For completeness, we also report here a 
modification in the vector potential. Indeed, the definitions (13)-(21) and the transversal components of (27) remain unchanged when we introduce a correction in the longitudinal component of A to obtain the so-called 2D3V model (Fig. 2)

$$
A_{z}\left(\mathbf{x}_{i}\right)=-\frac{1}{c} \sum_{j \neq i} q_{j} v_{z j} \log \left(r_{i j}^{2}+\varepsilon^{2}\right)=\hat{A}_{z}\left(\mathbf{x}_{i}\right) .
$$

We employ this model in Sec. V, where we perform the validation of the model for a beam-plasma interaction.

\section{A. Field approximation}

We have derived the analytic solution of the scalar and vector potentials, consistent with a finite-sized particle shape suitable for dynamic N-body simulations, but it turns out that the computational cost needed to compute the pair particle interaction is $O\left(N^{2}\right)$, where $N$ is the number of particles. ${ }^{19,20}$ Since we strive for a large number of particles for good statistics, a direct approach is computationally inefficient. However, we can have a good approximation of the fields using, for instance, a Barnes-Hut tree algorithm. This algorithm yields an approximate solution with a reasonable computational $\operatorname{cost} O(N \log N)$ and with controlled errors.

The Barnes-Hut tree algorithm is extensively described elsewhere, ${ }^{12,19,20}$ but for completeness, we provide a brief description here. The first step consists of introducing a data structure (quad-tree in 2D; oct-tree in 3D), where each node of the tree may contain one or more particles within a square box of side $s$. To approximate the summations, a so-called multipole acceptance criterion (MAC) is used to systematically group distant particles together into pseudoparticles (Fig. 3). One particularly intuitive MAC is the original Barnes-Hut criterion

$$
\theta_{c}=s / d<\theta
$$

For opening angles $s / d$ smaller than $\theta$, the pseudoparticle cluster is included in the local sum, whereas for larger angles, the node is subdivided into its child nodes, which are then recursively retested. Clearly, the larger the choice of $\theta$,

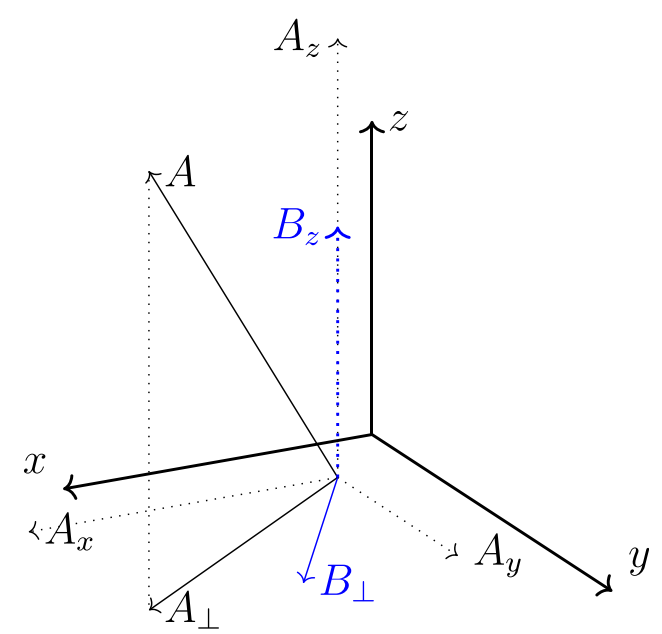

FIG. 2. Vector potential, schematic visualisation of the modified fields in the $2 \mathrm{D} 3 \mathrm{~V}$ geometry. It allows the development of a current along the $\mathrm{z}$ axis which yields a magnetic field in the plane $x-y$.

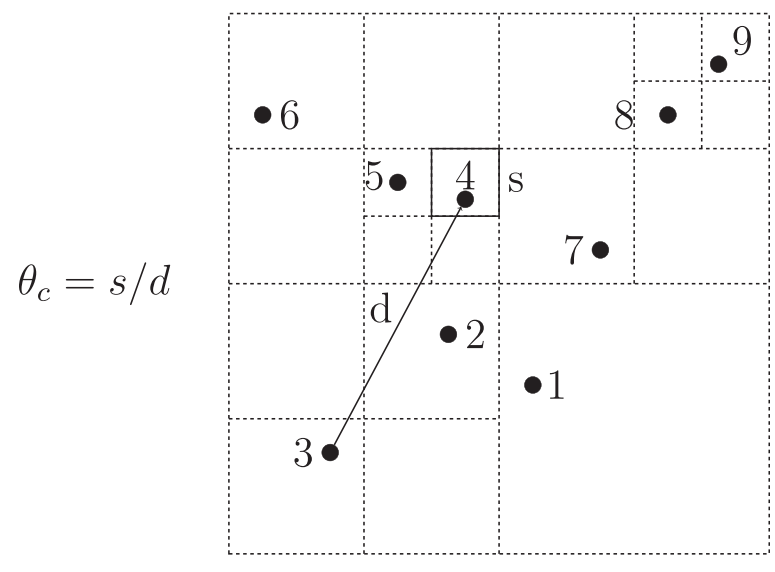

FIG. 3. Barnes-Hut acceptance criteria.

the faster the force summation will proceed at the cost of a larger error. A good compromise for $\theta$ lies within the interval $[0.3,0.7]$, which will yield errors well below $1 \%$, as we show later in Sec. V.

In order to improve the accuracy of the summations, we have calculated the Taylor expansion of the far fields (interaction particle-pseudoparticle) up to the second order. The present Darwin model is implemented within the parallel treecode framework PEPC. ${ }^{17,18}$

\section{HAMILTONIAN FORMULATION}

The present mesh-free formulation of the Darwin model avoids the usual Lagrangian formulation of the Lorentz equation. As already mentioned in other articles, ${ }^{1,7}$ this approach normally leads to numerical instabilities. As already explained, due to the Darwin approximation, we cannot use $\mathbf{E}^{s o l}=-\partial \mathbf{A} / \partial t$ in the Lorentz equation, because we would implicitly re-introduce retardations that we have neglected in Ampère law. Hence, a Lagrangian formulation is no longer consistent in this context. One way around this is to employ instead a Hamiltonian formulation of the Lorentz equation

$$
\frac{d \mathbf{p}_{i}}{d t}=q_{i}\left[\mathbf{E}^{i r r}\left(\mathbf{x}_{i}\right)+\mathbf{E}^{s o l}\left(\mathbf{x}_{i}\right)+\frac{1}{c} \mathbf{v}_{i} \times \mathbf{B}\left(\mathbf{x}_{i}\right)\right] .
$$

We indicate with $\mathbf{p}_{i}=m_{i} \gamma_{i} \mathbf{v}_{i}$ the momentum of the i-th particle. The Lagrangian at the coordinate $\mathbf{x}_{i}$ of the i-th particle subjected to an external electromagnetic field is

$$
\mathcal{L}_{i}=-\frac{m_{i} c^{2}}{\gamma_{i}}-q_{i} \varphi\left(\mathbf{x}_{i}\right)+\frac{q_{i}}{c} \mathbf{v}_{i} \cdot \mathbf{A}\left(\mathbf{x}_{i}\right) .
$$

We also define the generalized canonical momentum of the i-th particle, associated to the Lagrangian $\mathcal{L}_{i}$ as

$$
\mathbf{P}_{i}=\frac{\partial \mathcal{L}_{i}}{\partial \mathbf{v}_{i}}=m_{i} \gamma_{i} \mathbf{v}_{i}+\frac{q_{i}}{c} \mathbf{A}\left(\mathbf{x}_{i}\right) .
$$

In terms of the canonical momentum, we compute the Hamiltonian associated with the i-th particle as

$$
\mathcal{H}_{i}\left(\mathbf{x}_{i}, \mathbf{P}_{i}\right)=\mathbf{P}_{i} \cdot \mathbf{v}_{i}-\mathcal{L}_{i}=m_{i} \gamma_{i} c^{2}+q_{i} \varphi\left(\mathbf{x}_{i}\right)
$$


We then obtain the equations which govern the motion of the particles, subjected to magnetoinductive forces ${ }^{1}$

$$
\begin{gathered}
\dot{\mathbf{x}}_{i}=\frac{\partial \mathcal{H}_{i}}{\partial \mathbf{P}_{i}}=\frac{1}{m_{i} \gamma_{i}}\left[\mathbf{P}_{i}-\frac{q_{i}}{c} \mathbf{A}\left(\mathbf{x}_{i}\right)\right]=\mathbf{v}_{i}, \\
\dot{\mathbf{P}}_{i}=-\frac{\partial \mathcal{H}_{i}}{\partial \mathbf{x}_{i}}=-q_{i} \nabla \varphi\left(\mathbf{x}_{i}\right)+\frac{q_{i}}{c} \nabla\left(\mathbf{A}\left(\mathbf{x}_{i}\right) \cdot \mathbf{v}_{i}\right) .
\end{gathered}
$$

Similarly to what we have done for a single particle, we define the Lagrangian and Hamiltonian for a system of mutually interacting particles

$$
\begin{aligned}
\mathcal{L} & =\sum_{i}\left[-\frac{m_{i} c^{2}}{\gamma_{i}}-\frac{1}{2} q_{i} \varphi\left(\mathbf{x}_{i}\right)+\frac{1}{2 c} q_{i} \mathbf{v}_{i} \cdot \mathbf{A}\left(\mathbf{x}_{i}\right)\right] \\
\mathcal{H} & =\sum_{i} \mathbf{P}_{i} \cdot \mathbf{v}_{i}-\mathcal{L} \\
& =\sum_{i} m_{i} \gamma_{i} c^{2}+\frac{1}{2} \sum_{i} q_{i} \varphi\left(\mathbf{x}_{i}\right)+\frac{1}{2 c} \sum_{i} q_{i} \mathbf{v}_{i} \cdot \mathbf{A}\left(\mathbf{x}_{i}\right) .
\end{aligned}
$$

As mentioned before, the Hamiltonian formulation requires the adoption of an implicit integration scheme due to the correlation between the canonical momentum and the vector potential; in other words, the Hamiltonian is not fully separable

$$
\mathcal{H}_{i}\left(\mathbf{x}_{i}, \mathbf{P}_{i}\right)=\mathcal{T}_{i}\left(\mathbf{x}_{i}, \mathbf{P}_{i}\right)+\mathcal{V}_{i}\left(\mathbf{x}_{i}\right)
$$

where $\mathcal{T}_{i}, \mathcal{V}_{i}$ are, respectively, the kinetic and potential energies of the i-th particle.

The numerical scheme we introduce is effectively a semi-implicit Asymmetrical Euler method (AEM), which is a first order in time scheme, which consists of taking explicit velocity and implicit coordinate. This choice is motivated by the fact that if the vector potential becomes too strong, an implicit velocity in the numerical scheme may lead to numerical instability. In fact, under the condition of a strong vector potential, we might simplify Eq. (37)

$$
\dot{\mathbf{P}}_{i}=\frac{d}{d t}\left(\gamma_{i} \frac{d \mathbf{x}_{i}}{d t}\right)+\frac{q_{i}}{c} \frac{d \mathbf{A}_{i}}{d t} \approx 0 \approx \gamma_{i} \frac{d^{2} \mathbf{x}_{i}}{d t^{2}}+\beta \frac{d \mathbf{x}_{i}}{d t} .
$$

Necessarily, $\beta \gg \gamma_{i}$, and discretizing Eq. (41) with a second order centered scheme leads to numerical instabilities. According to Ref. 22, it is possible to prevent the formation of numerical instabilities by discretizing the second term in Eq. (41) with a backward scheme. Consequently, this trick implies the choice of taking explicit velocity in the discretized Hamiltonian Eqs. (36) and (37), which thus becomes

$$
\begin{gathered}
\mathbf{x}_{i}^{n+1}=\mathbf{x}_{i}^{n}+\Delta t \mathbf{v}_{i}^{n}, \\
\mathbf{P}_{i}^{n+1}=\mathbf{P}_{i}^{n}+q_{i} \Delta t\left[-\nabla \varphi_{i}^{n+1}+\frac{1}{c} \nabla\left(\mathbf{A}_{i}^{n+1} \cdot \mathbf{v}_{i}^{n}\right)\right] .
\end{gathered}
$$

Therefore, Eq. (42) is not coupled to the momentum equation, Eq. (43), rather it is given explicitly. This implies that Eq. (43) might be computed in an explicit fashion, by updating the fields at the new particles' positions. The method presented is a good compromise between computational costs and conservation of the first integrals of the motion. On the other hand, there is also ample scope for improving the latter by adopting higher order time integration schemes. ${ }^{27}$

\section{NUMERICAL RESULTS}

In this section, we focus our attention on the validation of the model described in Secs. III and IV. The validation is organized in four physical test cases: the first is a static electron beam with cylindrical symmetry. The second test is a simple Langmuir wave with the magnetic field being neglected. This is followed by a dynamic test involving both electrostatic and magnetic fields using the expansion of cylindrically symmetric electron beam. Finally, we examine the filamentation instability of an electron beam penetrating the plasma.

\section{A. Static electron beam}

The first test case we consider is a static electron beam with uniform density in a disc, where an analytic solution is known. ${ }^{23}$ The goals of this test are to study the precision of the field solver. Two calculations are shown, N1 and N2, respectively, with $4 \times 10^{4}$ and $25 \times 10^{4}$ particles distributed in a disc having radius $r_{0}=0.2 c / \omega_{p e}$. The beam has an initial longitudinal velocity $v_{z}=0.4 \mathrm{c}$. We compare the electric and magnetic fields and current density with the corresponding theoretical solution. Given a current $I=n v_{z} \pi r_{0}^{2}$, for the above configuration, the theoretical current density is then given by

$$
J_{z}(r)=\frac{I}{r_{0}^{2} \pi} \quad \text { for } \quad 0 \leq r \leq r_{0} .
$$

By inverting the Gauss law, it turns out that the electric field has only a radial component

$$
\begin{array}{cc}
E_{r}(r)=\frac{I r}{2 \pi \epsilon_{0} r_{0}^{2} v_{z}} \quad \text { for } \quad 0 \leq r \leq r_{0}, \\
E_{r}(r)=\frac{I}{2 \pi \epsilon_{0} v_{z} r} \quad \text { for } \quad r \geq r_{0} .
\end{array}
$$

Similarly, we derive the magnetic field from Ampère's law, which has only an azimuthal component

$$
\begin{gathered}
B_{\theta}(r)=\frac{\mu_{0} I r}{2 \pi r_{0}^{2}} \quad \text { for } \quad 0 \leq r \leq r_{0}, \\
B_{\theta}(r)=\frac{\mu_{0} I}{2 \pi r} \quad \text { for } \quad r \geq r_{0} .
\end{gathered}
$$

The simulation performed is in good agreement with the analytic solution as shown in the panels of Figs. 4-6. The better accuracy in the simulation $\mathrm{N} 2$ is obtained by virtue of the improved statistics.

To check the accuracy and convergence of the BarnesHut tree algorithm for the full set of magnetoinductive fields, the relative error in the vectorial field of interest is compared with the directly computed pair-particle solution. Therefore, 

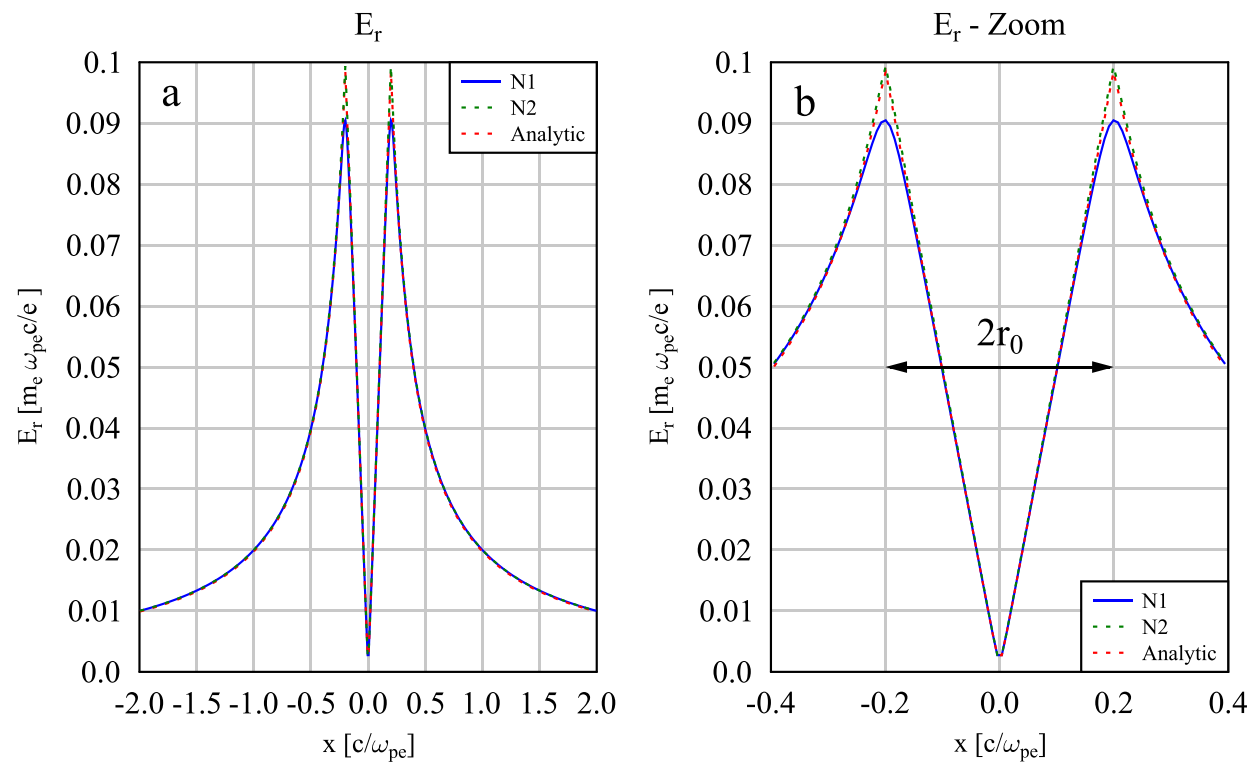

$\mathrm{B}_{\theta}$

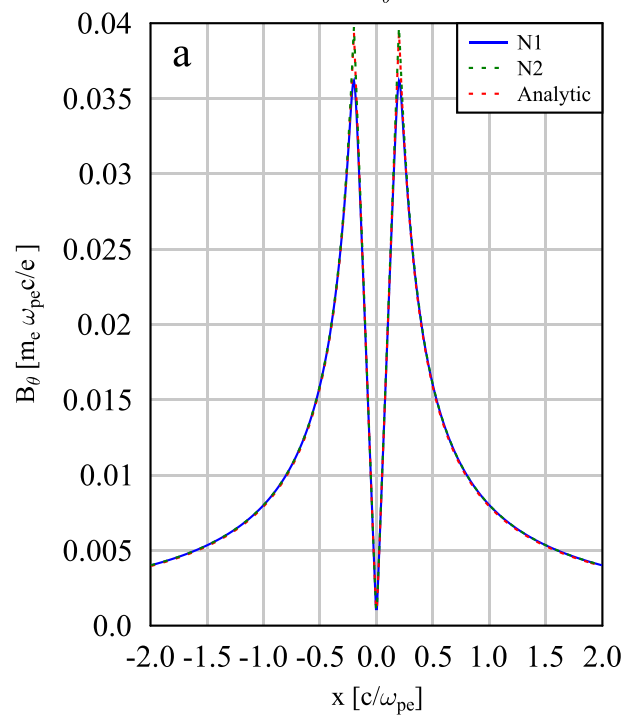

$\mathrm{J}_{\mathrm{Z}}$
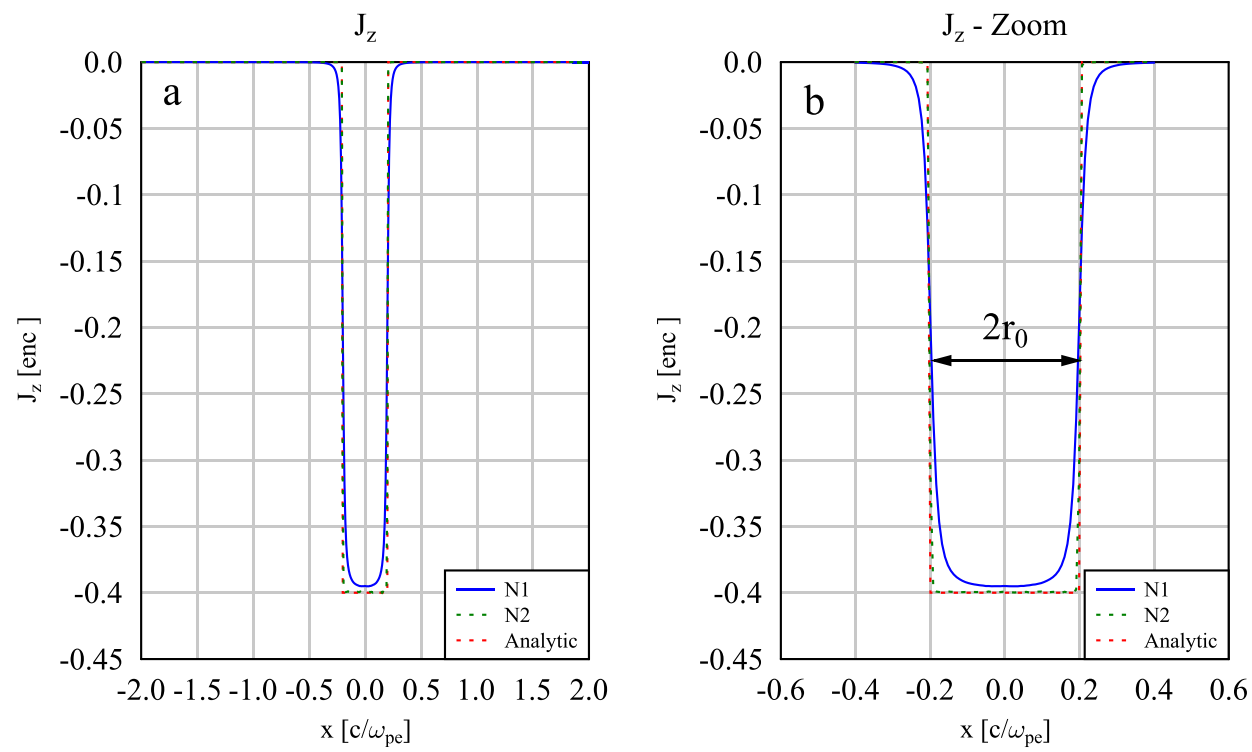

FIG. 4. Static electron beam. In the graphs, we show $E_{r}(x)$. We show two numerical solutions, the first with $4 \times 10^{4}$ particles (blue line, N1) and the second one with $25 \times 10^{4}$ particles (dotted green, N2), both present good agreement with the theoretical curve (dotted red).
FIG. 5. Static electron beam. In the graphs, we show $B_{\theta}(x)$. We show two numerical solutions, the first with $4 \times 10^{4}$ particles (blue line, N1) and the second one with $25 \times 10^{4}$ particles (dotted green, N2), both present good agreement with the theoretical curve (dotted red).

FIG. 6. Static electron beam. The current density is uniform. In the graphs, we show $J_{z}(x)$. We show two numerical solutions, the first one with $4 \times 10^{4}$ particles (blue line, N1) and the second one with $25 \times 10^{4}$ particles (dotted green, N2), both present good agreement with the theoretical curve (dotted red). 


\section{Relative Error in J - E - B}

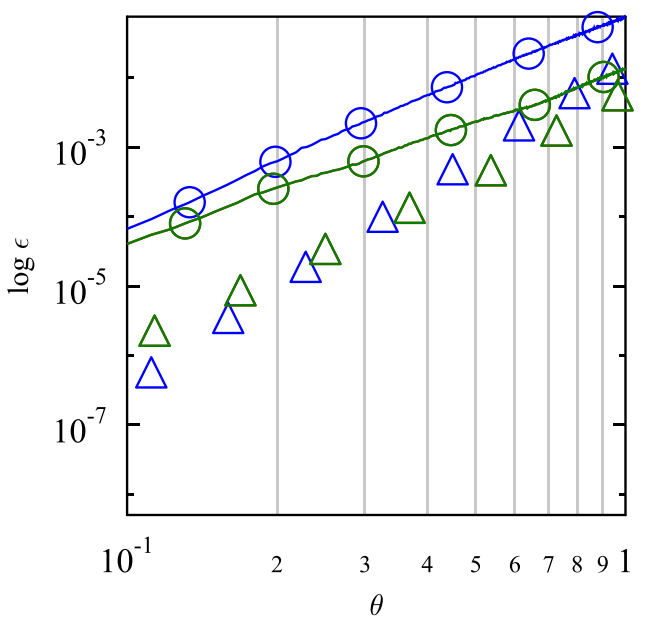

FIG. 7. Static electron beam. In the graph, we show relative errors for the monopole (circle), dipole (line) and quadrupole (triangle) with respect to the pair particle fields. The relative errors are computed for $J(x), E_{r}(x)$ and $B_{\theta}(x)$, which are colored, respectively, in blue, red and green. These errors grow for increasing MAC value. These simulations are performed with $10^{4}$ particles.

in Fig. 7, the respective relative errors incurred in the electric and magnetic fields and current density are shown. The relative error $\epsilon$ (for instance, in the electric field) is computed according to the formula

$$
\epsilon_{k}^{2}(\mathbf{E}, \theta)=\frac{\sum_{j=1}^{n}\left[\mathbf{E}_{j k}^{\theta}-\mathbf{E}_{j k}^{\theta=0}\right]^{2}}{\sum_{j=1}^{n}\left[\mathbf{E}_{j k}^{\theta=0}\right]^{2}},
$$

where the index $k$ stands for the order of Taylor expansion, $\theta$ is the MAC number $(\theta=0$ means pair-particle interaction) and $n$ is the number of particles. The previous figures exhibit two important facts. First, we can see that the relative errors in all three quantities decrease as we increase the order of the Taylor expansion. Second, the relative errors have an exponential trend; furthermore, we have an estimation of the error once we set the multipole angle criteria. An additional confirmation of the good results obtained lies in the equivalence of the error's curves between the monopole and the dipole; indeed, the uniform mass and velocity in the initial configuration imply a vanishing dipole contribution. Furthermore, the relative error of the magnetic field is exactly the same as the relative error of the electric field as expected; in fact, in this configuration, the velocity is constant.

\section{B. Langmuir wave}

While in the first test case we have looked at a static benchmark, in the second test, we simulate a Langmuir wave. The purpose of this benchmark is to validate the integration scheme with pure electrostatics before tackling a more complex truly magnetoinductive case, making a comparison with the explicit Leap-Frog scheme, ${ }^{21}$ in fact, for electrostatic problems, a full explicit scheme is a good compromise between computational effort and relative errors. The explicit Leap-Frog for electrostatic problems is

$$
\begin{gathered}
\mathbf{x}_{i}^{n+1}=\mathbf{x}_{i}^{n}+\Delta t \mathbf{v}_{i}^{n}, \\
\mathbf{v}_{i}^{n+1}=\mathbf{v}_{i}^{n}-\frac{q_{i}}{m_{i}} \Delta t \nabla \varphi_{i}^{n} .
\end{gathered}
$$

Here, electrons oscillate around stationary ions. The simulations performed have the following initial parameters: $n=4 \times 10^{6}$ as the total number of particles, the ions are at rest, while the electrons have a Boltzmann-Maxwell distribution with $v_{t h}=0.05 \mathrm{c}$. A small perturbation is introduced in the electron velocity distribution, $\alpha=0.05$ according to the formula

$$
f_{e}(0, \mathbf{x}, \mathbf{v})=\frac{1}{2 \pi}\left(1+\alpha \cos \left(k_{x} x\right)\right) e^{-v^{2} / 2 v_{t h}} .
$$

We choose as the time step/ $\omega_{p e} \Delta t=0.1$ and the final time $\omega_{p e} t=30$. The rectangular $[0,2 \pi] \times[0, \pi]\left(c / \omega_{p e}\right)^{2}$ is used as a domain and we employ periodic boundary conditions.

In this benchmark, we resolve the plasma frequency of a plasma. From theory, we expect that the electric energy along the $\mathrm{x}$-axis has a frequency $\omega=1$

$$
E_{x}^{2}=\sum_{i}\left(E_{x}\right)_{i}^{2}
$$

It is possible to observe the Fourier spectrum of electric energy, Fig. 8, showing good agreement with theory and revealing plasma wave harmonics even at this relatively low amplitude, a testament to the low-noise characteristics of the grid-free approach.

In the electrostatic case, a Leap-Frog integrator is widely used, since its computational effort is much lower than an implicit scheme and it produces acceptable relative error in the total energy. The energy diagram is shown in the following graph, it is meant to compare the relative error in the total energy between a Leap-Frog and the integrator introduced in Sec. IV

We see from Fig. 9 that in all cases energy is conserved to around $1 \%$ over ten plasma periods. For the larger time step, the system continues to heat with the Leap-Frog

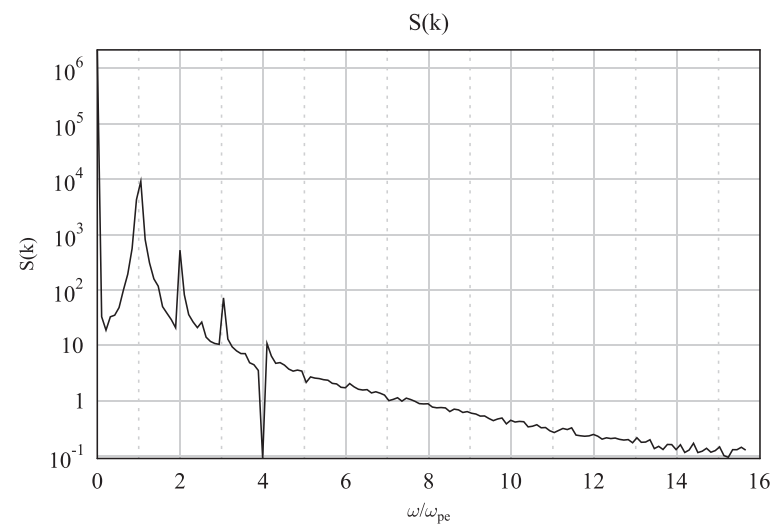

FIG. 8. Langmuir wave in cold plasma, Fourier analysis of the electric energy, the maximum shows the main frequency. 


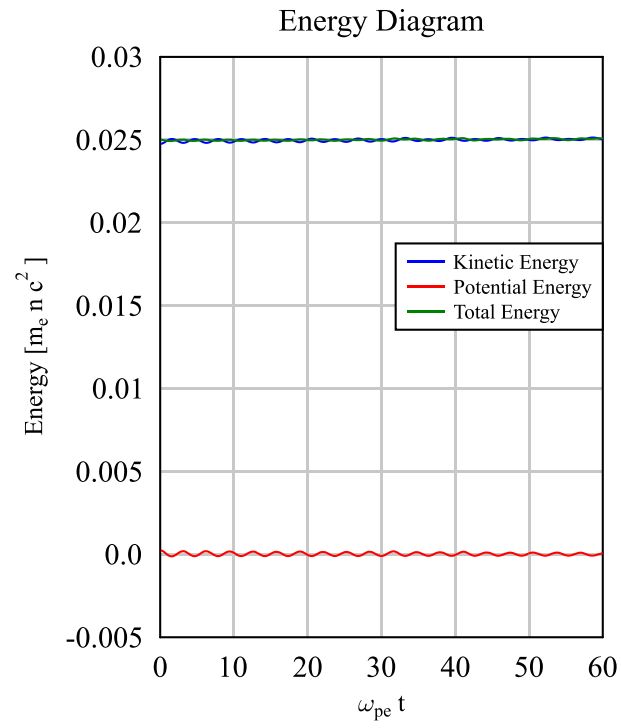

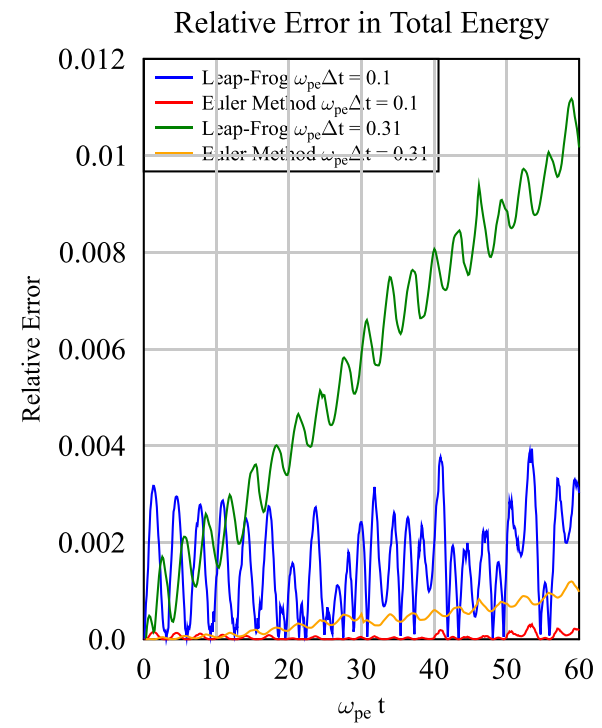

FIG. 9. Langmuir wave. Energy diagram on the left panel and a comparison of relative errors between LeapFrog and Asymmetrical Euler Method (AEM) on the right panel, for two different time steps, $\omega_{p e} \Delta t=0.1,0.31$. scheme and less with the AEM, indicating that energy conservation is sensitive to the integration scheme. In Ref. 28, Huang et al. have recently discussed numerical heating in PIC codes resulting from finite-grid instability (FGI), identifying the lack of spectral fidelity (i.e., neglect of shortwavelength modes) in the density deposition and field interpolation as the major culprit-an effect which does not arise in our method. We note in passing that the "gridless" method used by Huang et al. for comparison relied on a discretised representation of the density and the electric field in Fourier $(k-)$ space, and as such differs fundamentally from the realspace field-solvers used in our algorithm.

\section{Electron beam}

We now present a first real, dynamic test of the Darwin model. The setup we have chosen is an electron beam propagating through vacuum. The geometry is a disk as the static electron beam has shown previously. According to Ref. 24, it is possible to estimate the expansion of the RMS radius and the transversal velocity

$$
\begin{aligned}
& R^{2}=\frac{1}{n} \sum_{i=1}^{n} r_{i}^{2} \quad V^{2}=\frac{1}{n} \sum_{i=1}^{n} v_{i}^{2}, \\
& \ddot{R}-\frac{U_{b}}{\beta^{2} \gamma^{2} R}=\frac{\left[R^{2}\left(V^{2}-\dot{R}^{2}\right)\right]_{t=t_{0}}}{R^{3}},
\end{aligned}
$$

where $U_{b}=q \beta c \mu_{0} I_{b} / 4 \pi \gamma m=\left(\beta c^{2} / \gamma\right) I_{b} / I_{0}$ and $I_{0}$ is the Alfvén critical current. To derive this estimate, it is assumed that the perveance of the electron beam is much smaller than one, that is, its transversal velocity is negligible with respect to the longitudinal component. We have carried out a simulation employing $10^{5}$ electrons distributed in a disk having radius $r_{0}=0.2 c / \omega_{p e}$. The particles have a longitudinal velocity $\gamma v_{z} / c=\gamma \beta=0.8$; we also assume that there is no initial transverse motion. The initial configuration yields the following perveance: ${ }^{23}$

$$
K=\frac{2 I_{b}}{I_{0} \beta^{3} \gamma^{3}}=\frac{1}{2 \gamma}\left(\frac{\omega_{p e} r / c}{\beta \gamma}\right)^{2} \simeq 2.4 \times 10^{-2},
$$

consistent with the assumptions made in the analytical model.
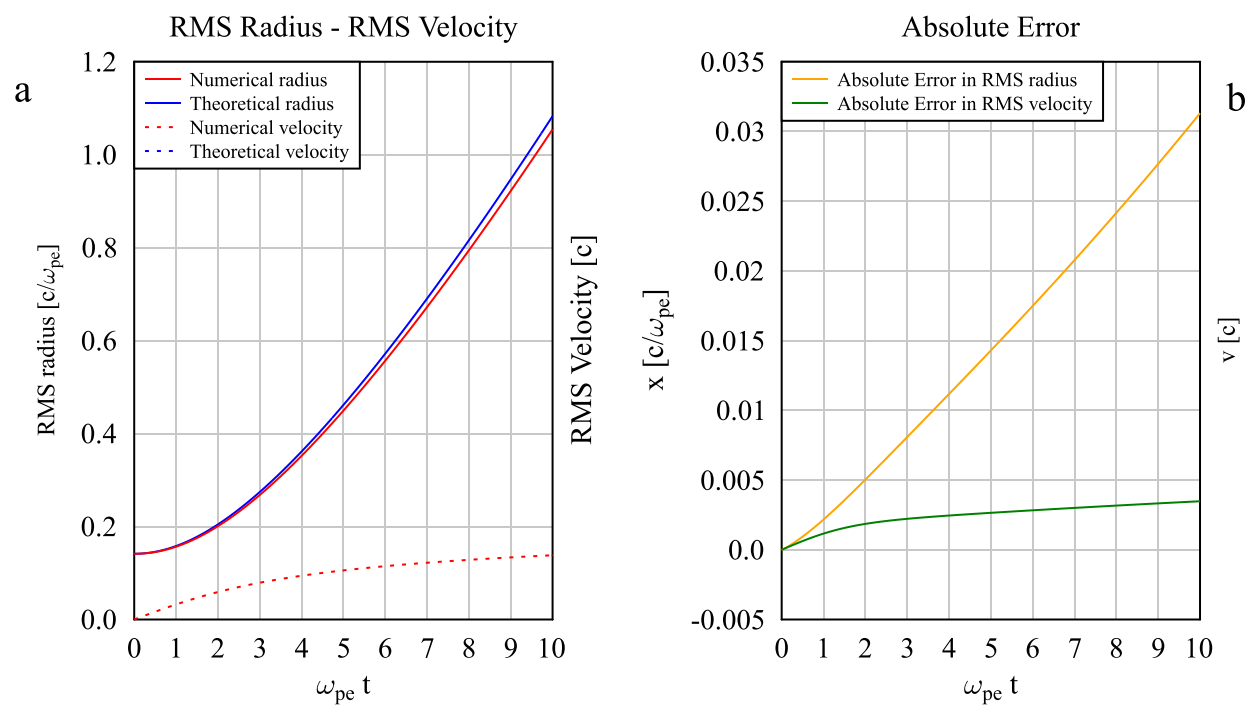

FIG. 10. Electron Beam. In panel (a) we compare the theoretical RMS radius (blue line) with the numerical RMS radius (red line). The dotted lines are the theoretical RMS transversal velocity (blue) and the numerical RMS transversal velocity (red). We have performed an analysis of the absolute error computed in panel (b). 


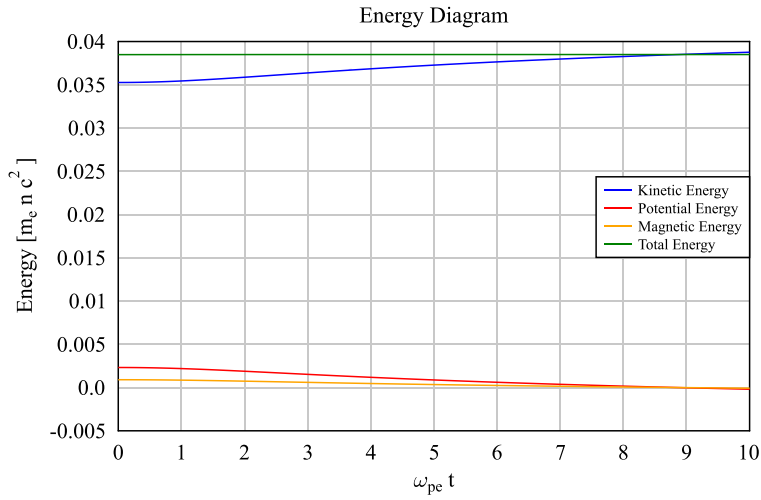

FIG. 11. Electron Beam, the above energy diagram shows kinetic energy in blue, potential energy in red, magnetic energy in orange and total energy in green.

Figure 10 shows good agreement with the theory in both RMS radius and transverse velocity. Further simulations with up to $5 \times 10^{5}$ electrons show no significant change in the numerical results. The slight departure from the analytical result may be due to the envelope equation not taking into account the longitudinal interaction among particles, and secondly, the transversal velocities becoming relativistic, which would violate the initial hypothesis $\left(\left\langle\mathbf{v}_{\perp}\right\rangle \ll v_{z}\right)$. Despite the fact that the scheme does not guarantee conservation of the prime integrals $\left(\mathcal{H}, P_{x}, P_{y}\right.$ and $\left.P_{z}\right)$, we still achieve a good accuracy in the relative errors (Fig. 11).

We have also performed a parametric study by varying the MAC value, indicated by $\theta$ in the following Figs. 12 and 13. The relative errors are defined at time step $\mathrm{k}$, for instance, in the total energy, as follows:

$$
\epsilon_{\text {rel }}^{k}=\frac{\left|\mathcal{H}^{k}-\mathcal{H}^{0}\right|}{\mathcal{H}^{0}},
$$

while the maximum error in the canonical momenta are defined at time step $\mathrm{k}$ as

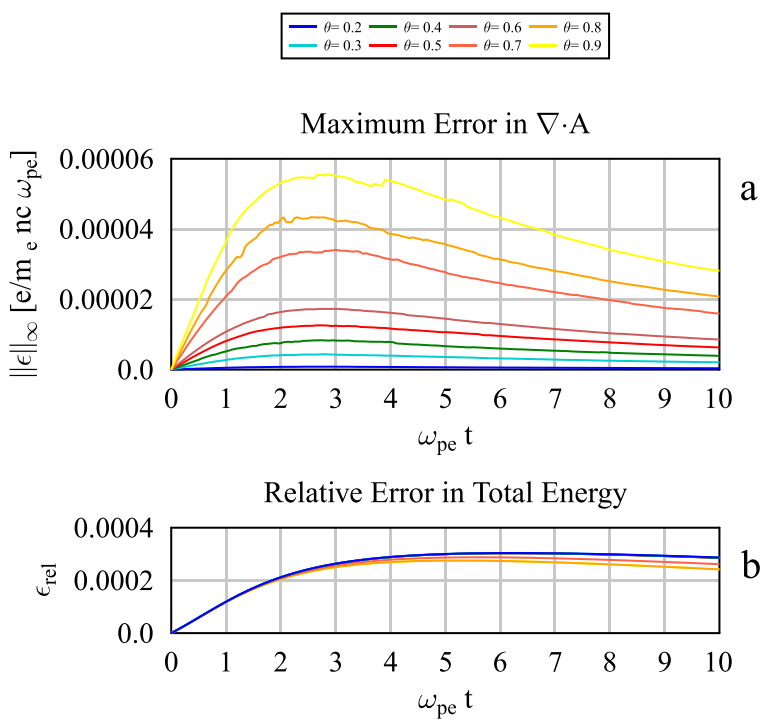

FIG. 12. Electron Beam. A parametric study of the MAC value $(\theta)$. The top panel (a) exhibits the maximum errors in $\nabla \cdot \mathbf{A}$, while panel (b) shows the relative error in the total energy.

$$
\|\epsilon\|_{\infty}^{k}=\max _{i}\left\{\left|\mathbf{P}_{i}^{k}-\mathbf{P}_{i}^{k-1}\right|\right\} .
$$

It is worth mentioning that the divergence of the vector potential $\nabla \cdot \mathbf{A}$ converges to zero when $\theta$ tends to zero; this behavior is highlighted in Fig. 12, where panel (a) exhibits the maximum error in $\nabla \cdot \mathbf{A}$. In contrast to most Darwin PIC codes, no divergence cleaning is needed for the present method. The parametric study also shows how the relative error in total energy depends slightly on $\theta$, but not dramatically [panel (b)].

By contrast the errors in the momenta are independent of the MAC value, so the accuracy on the energy and momenta depend mainly on the choice of the time integration scheme.

\section{Weibel instability}

In the last test, we present a study of the Weibel instability in a beam-plasma system-such a configuration was first studied in Ref. 25. The motivation for this test lies in the generic nature of this phenomenon, in fact, it is a topic often encountered in both astrophysical (e.g., magnetic reconnection) and laboratory (e.g., fast ignition) plasmas. ${ }^{26} \mathrm{We}$ summarize the initial configuration: homogeneous, collisionless, charge- and current-neutral system. We study the transverse dynamics of two initially uniform currents, the former is a relativistic beam, whereas the second is a return current which guarantees initial charge and current neutrality. The plasma is characterized by density $n_{p 0}$, while the beam has density $n_{b 0} / n_{p 0}=0.1$ and Lorentz factor $\gamma_{b 0}=2.5$. We also assume that electrons have a Maxwellian distribution with thermal velocity $\left\langle v_{\perp}\right\rangle / v_{b}=10^{-4}$, while the ions are initially at rest. The simulated domain is a square having $20 \mathrm{c} / \omega_{p e}$

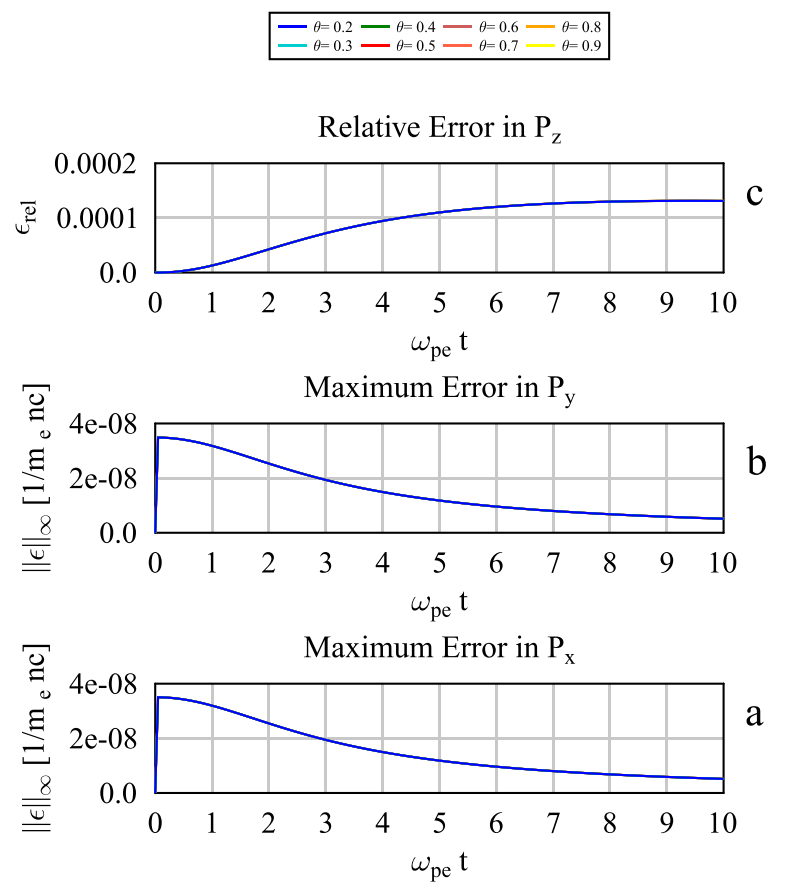

FIG. 13. Electron Beam. A parametric study of the MAC value $(\theta)$, panels (a) and (b) exhibit the maximum errors in the transversal canonical momenta, respectively, $\mathbf{P}_{x}$ and $\mathbf{P}_{y}$. Panel (c) shows the relative error and in the longitudinal momentum, $\mathbf{P}_{z}$. 


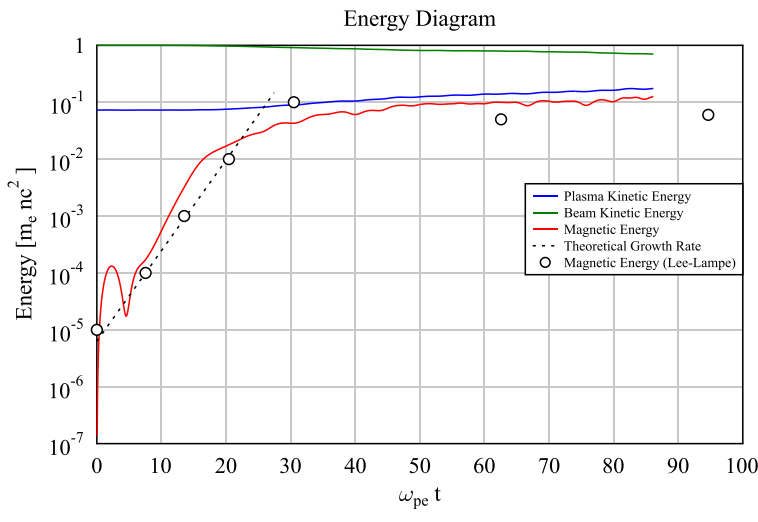

FIG. 14. Filamentation instability. Energy diagram in panel (a), it compares the electron beam and the plasma kinetic energy with magnetic energy, the quantities are in units of the initial beam energy. The dotted line shows the analytical growth rate according to Eq. (59).

size and $8 \times 10^{6}$ total number of particles. This is not intended to mock up any realistic physical system, but is closest to the periodic geometry used in the literature (open boundaries are used for our simulations).

According to Fig. 14, it is apparent that the relativistic beam electrons transfer energy and heat the plasma until non-linear mechanisms dominate the dynamics.

The magnetic energy exhibits an exponential behavior as predicted from the linear theory, under this circumstance, the dispersion relation is 25

$$
Z^{\prime}\left(\omega / k v_{\perp}\right)=-\left(\omega_{p}^{2}+k^{2} c^{2}\right) v_{\perp}^{-2}\left(v_{\perp} / v_{b}\right)^{2} .
$$

The linear regime is suppressed by a non-linear mechanism at about $\omega_{p e} t=20$. Figure 15 highlights good conservation of canonical momenta and divergence of $\mathbf{A}$ [panels (a) and (b), respectively], but as Ref. 21 points out, the time integration scheme used does not guarantee conservation of the total energy [panel (c) shows the relative error in the total energy]. At later times, as in Ref. 25 , the beam electrons start to attract each other due to magnetic interaction, and repel plasma electrons. Thus, the beam electrons coalesce into filaments which recombine and decrease in number.
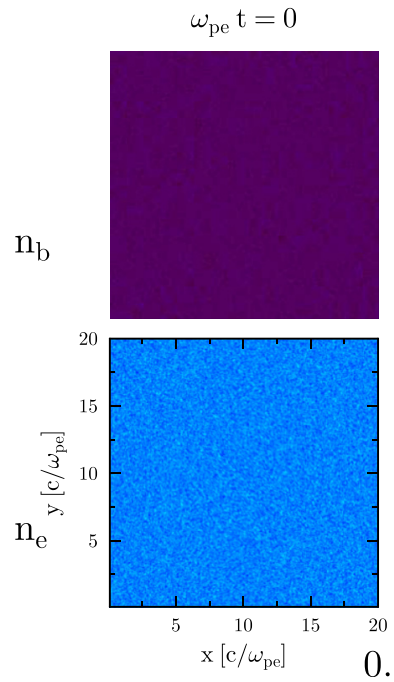

$\omega_{\text {pe }} \mathrm{t}=20$

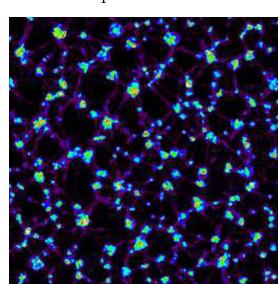

$\omega_{\text {pe }} \mathrm{t}=40$
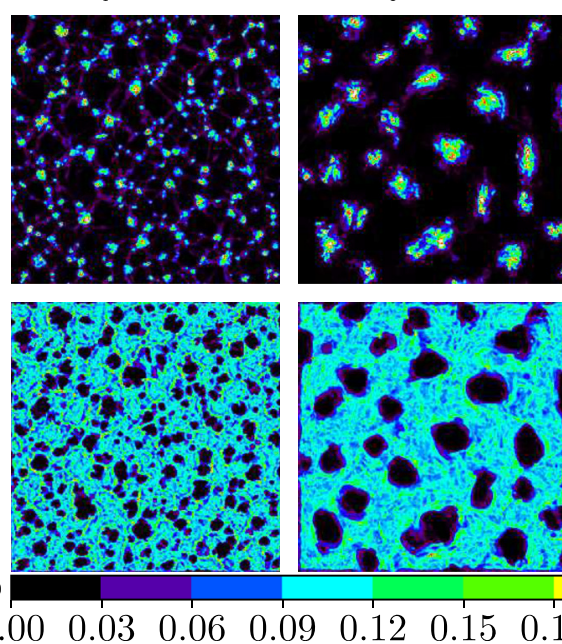

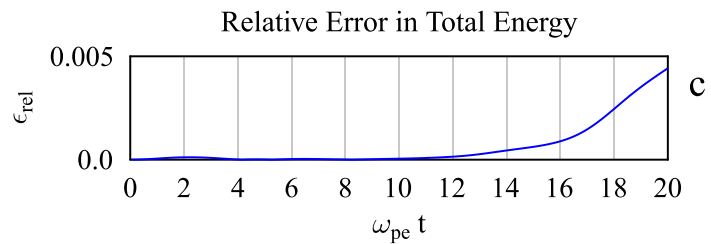

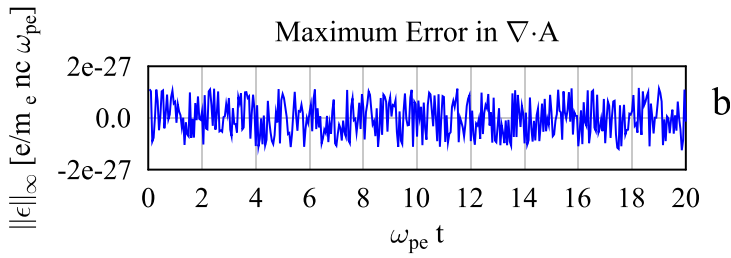

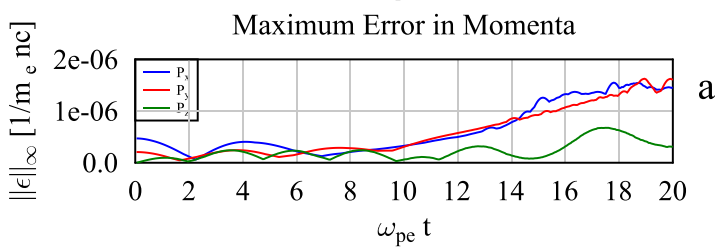

FIG. 15. Filamentation instability. Canonical momenta diagram in panel (a), it compares the maximum errors in $\mathbf{P}_{x}, \mathbf{P}_{y}$ and $\mathbf{P}_{z}$. Panel (b) exhibits the maximum error in $\nabla$. A. Finally, panel (c) shows the relative error in the total energy.

In Fig. 16, we show the beam ( $n_{b}$, first row) and plasma ( $n_{e}$, second row) electron density and clearly exhibit the coalescing process up to the point where the beam reaches the box size, or in our case, the same dimensions as the initial plasma region. According to Ref. 26, in the present configuration, the Alfvén critical current is always bigger than the forward current. In fact, the initial Alfven current is $I_{A}(t=0)=m_{e} c^{3} / e\left\langle\gamma v_{z} / c\right\rangle \simeq 2.291 m_{e} c^{3} / e$, whereas the maximum current contained within a filament at $\omega_{p e} t=60$ is $I_{p}^{\max } \simeq 0.28 m_{e} c^{3} / e$ for this example.

However, it is worth noting a discrepancy between the present simulation and Ref. 25, in fact, the recombination process of the filaments continue at a reduced speed, which is caused by the open boundary conditions. In this example, the particles were artificially arranged in a square box, but naturally tend to form a circular configuration over time. Additional simulations starting with circular beam/plasma geometry yielded similar results for the linear and non-linear phases.

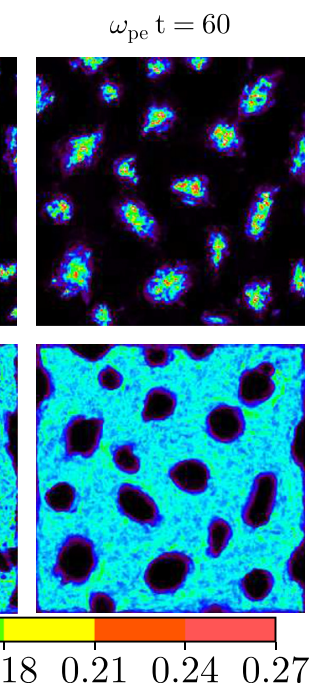

FIG. 16. Filamentation instability. Electron density in the plane $x-y$ at times indicated $\left(\omega_{p e} t=0,20,40,60\right)$. It exhibits the evolution of the beam electron density (first row $n_{b}$ ) and the plasma electron density (second row $n_{e}$ ). The densities are in units of $\max \left(n_{b}+n_{e}\right)$ 


\section{CONCLUSIONS}

To summarize, a new formulation of a Darwin model has been presented, based on a special formulation of scalar and vector potentials for two-dimensional, finite-sized particles. To avoid the well-known instability exhibited by explicit time integration, we have implemented a semiimplicit scheme based on a Hamiltonian formulation utilizing the canonical momenta as dynamic variables. Static benchmark tests show good agreement with the analytical theory, verifying the expected convergence of the field solver within the multipole approximation used. The time integration scheme has been compared with the explicit Leap-Frog for a simple electrostatic Langmuir plasma wave. The semiimplicit scheme achieves a better conservation in the total energy. A genuine magnetoinductive test of a relativistic electron beam propagating in a vacuum achieves very high accuracy and conservation of energy and momenta. Finally, a challenging test for the present model is shown, which despite the small differences in the boundary conditions applied, reproduces the essential findings obtained with fully electromagnetic PIC codes by Refs. 25 and 26. Further detailed studies would be needed to investigate the pros and cons of the present mesh-free approach over EM and Darwin PIC codes in the context of beam-plasma simulation-especially for more complex geometries.

Further improvements in conservation properties can be expected in future by incorporating a higher order time integration scheme such as Runge-Kutta or variational methods, which should also permit the use of time steps approaching the ion time-scale. Extensions to a fully 3D model should be straightforward by employing the vector potential already formulated in Ref. 7. The Darwin formulation described here avoids the necessity of a grid inherent to classical particlein-cell (PIC) approaches, and may open up new modeling possibilities for laser-irradiated plasmas (such as electron transport in fast ignition schemes), or magnetic reconnection and whistler waves in space plasmas.

\section{ACKNOWLEDGMENTS}

The simulations were performed with the computing resources granted by the VSR of the Research Centre Jülich under project JZAM04. This work was supported by Geconcerteerde Onderzoeksacties (GOA) from KU Leuven Bijzonder Onderzoeksfonds (BOF) and Helmholtz Association Grant HIRG-0048.

${ }^{1}$ C. W. Nielsen and H. R. Lewis, "Particle-code models in the nonradiative limit," Methods in Computational Physics (Academic Press, New York, 1976), Vol. 16, p. 367.

${ }^{2}$ D. W. Hewett, Space Sci. Rev. 42, 29-40 (1985).

${ }^{3}$ D. W. Hewett, Comput. Phys. Commun. 84, 243-277 (1994).

${ }^{4}$ N. Besse, N. Mauser, and E. Sonnendrücker, J. Appl. Math. Comput. Sci. 17, 3 (2007).

${ }^{5}$ H. Schmitz and R. Grauer, J. Comput. Phys. 214, 738-756 (2006).

${ }^{6} \mathrm{G}$. Chen and L. Chacón, Comput. Phys. Commun. 185, 10 (2014).

${ }^{7}$ M. Mašek and P. Gibbon, IEEE Trans. Plasma Sci. 38(9), 2377 (2010).

${ }^{8}$ A. J. Christlieb, R. Krasny, J. P. Verboncoeur, J. W. Emhoff, and I. D. Boyd, IEEE Trans. Plasma Sci. 34(2), 149 (2006).

${ }^{9}$ T. B. Krause, A. Apte, and P. J. Morrison, Phys. Plasmas 14, 102112 (2007).

${ }^{10}$ J. D. Jackson, Classical Electrodynamics, 2nd ed. (Wiley, New York, 1975).

${ }^{11}$ L. D. Landau and E. M. Lifshitz, The Classical Theory of Fields (Butterworth-Heinemann, 1987).

${ }^{12}$ L. Hernquist and J. E. Barnes, Nature 354, 210-212 (1991).

${ }^{13} \mathrm{G}$. S. Winckelmans, "Topics in vortex methods for computation of threeand two-dimensional incompressible unsteady flows," Ph.D. thesis (California Institute of Technology, Pasadena, California, 1989).

${ }^{14}$ P. Gibbon, Phys. Rev. E 72, 026411 (2005).

${ }^{15}$ B. Steinbusch, P. Gibbon, and R. D. Sydora, Phys. Plasmas 23, 052119 (2016).

${ }^{16}$ M. Winkel and P. Gibbon, Contrib. Plasma Phys. 53, 254 (2013).

${ }^{17}$ P. Gibbon, R. Speck, A. Karmakar, L. Arnold, W. Frings, B. Berberich, D. Reiter, and M. Mašek, IEEE Trans. Plasma Sci. 38(9), 2367 (2010).

${ }^{18}$ M. Winkel, R. Speck, H. Hübner, L. Arnold, R. Krause, and P. Gibbon, Comput. Phys. Commun. 183(4), 880 (2012).

${ }^{19}$ S. Pfalzner and P. Gibbon, Many Body Tree Methods in Physics (Cambridge University Press, 1996).

${ }^{20}$ J. Barnes and P. Hut, Nature 324, 446 (1986).

${ }^{21}$ B. Leimkuhler and S. Reich, Simulating Hamiltonian Dynamics (Cambridge University Press, 2005).

${ }^{22}$ A. Quarteroni, Numerical Models for Differential Problems (Springer, 2010).

${ }^{23} \mathrm{M}$. Reiser, Theory and Design of Charged Particle Beams (Wiley, 2008).

${ }^{24}$ E. P. Lee and R. K. Cooper, Part. Accel. 7, 83-95 (1976).

${ }^{25}$ R. Lee and M. Lampe, Phys. Rev. Lett. 31, 1390 (1973).

${ }^{26}$ M. Honda, J. Meyer-ter-Vehn, and A. Pukhov, Phys. Plasmas 7(4), 1302 (2000).

${ }^{27}$ S. A. Chin, Phys. Rev. E 80, 037701 (2009).

${ }^{28}$ C. K. Huang, Y. Zeng, Y. Wang, M. D. Meyers, S. Yi, and B. J. Albright, Comput. Phys. Commun. 207, 123-135 (2016). 